\title{
Maximum Power Control of Gyroscopic Wave Energy Converter
}

\author{
Lisheng Chen ${ }^{1}$, Zhongcai Pei ${ }^{1}$, Zhiyong Tang ${ }^{1}$ \\ ${ }^{1}$ School of Automation Science and Electrical Engineering, Beihang University, Beijing, China
}

\begin{abstract}
A new type of wave energy converter device, gyroscopic wave energy converter (GWEC) based on gyroscopic precession principle, is proposed in this paper. The dynamic model of the device is established, and a variable load maximum power point tracking (MPPT) method is adopted to achieve the goal of keeping the system near the maximum power when the wave amplitude changes. The maximum power point of the circuit is found by perturbation and observation method and incremental conductance method to form a specified PWM wave, which can control the switching on and off, then change the load of the circuit, and realizes the maximum power tracking of the system. Compared with the simulation results, the two methods have their own characteristics, but they can basically adjust the equivalent load adaptively with the change of wave amplitude, and achieve the maximum power output of the circuit.
\end{abstract}

\section{Introduction}

With the development of society and economy, the exhaustion of traditional energy sources, such as fossil fuels, is becoming more and more serious. Renewable energy comes into being at the historic moment and is attracting more and more people's attention and favor. Ocean energy is abundant in different renewable energy sources, and wave energy in ocean energy has the least damage to the environment in the process of development and utilization. Therefore, many countries in the world have carried out research on wave energy power generation technology. In recent decades, many types of wave power generation devices have been developed, but the technology of wave power generation is not yet fully mature, and many engineering problems need to be studied [1].

Wave Energy Converter (WEC) can be divided into air pressure type, hydraulic type, mechanical type and direct drive type according to the form of wave energy capture. The common air pressure WEC is the oscillating water column type. When the waves rise and fall, compressed air drives the steam turbine to generate electricity [2]. Hydraulic WEC can be divided into pendulum type and raft type. Wave energy is used to drive hydraulic device and hydraulic turbine is used to drive generator to generate electricity [3]. Mechanical WEC converts wave energy into rotating mechanical energy by driving shaft and gear box to generate electricity. Directdrive WEC usually uses linear motor to directly convert mechanical energy of wave into electric energy, eliminating intermediate conversion link, improving conversion efficiency and reducing maintenance cost [4].

For the power generation control of wave energy converter, Hugo Mendonca et al. simulated the change of load resistance value through three-phase boost rectifier circuit, and verified that there is an optimum load resistance value corresponding to a specific wave period and amplitude, so as to maximize the power generation of wave energy replacement device, which provides a good control idea for the control research of direct drive device [5].In addition, Amon et al. proposed the structure of uncontrollable rectifier bridge plus buck converter. By adjusting the duty cycle of Buck circuit, we can find the optimal value suitable for the current wave environment. But the uncontrollable rectifier bridge will make the current harmonic content larger [6].

In this paper, a kind of wave energy converter based on gyro precession effect is proposed, and two maximum power point tracking algorithms are used to keep the circuit running near the maximum power.

\section{Gyroscopic wave energy converter}

The gyroscopic wave energy converter studied in this paper is mainly composed of gyroscopic flywheel chamber, floating platform, gear transmission and a generator. Its structure is shown in Figure 1.

When the wave acts on the floating platform, the platform will generate swing angular velocity. The gyroscope mechanism and the floating platform are fixed together to swing. The gyroscopic mechanism obtains the swing angular velocity while its flywheel rotates at high speed, which causes the gyroscopic mechanism to generate precession and torque on the moving shaft. The rotational speed is increased by gear transmission, and the torque is transmitted to the generator to generate electricity.

\footnotetext{
* Corresponding author: Ethanchen33@163.com
} 


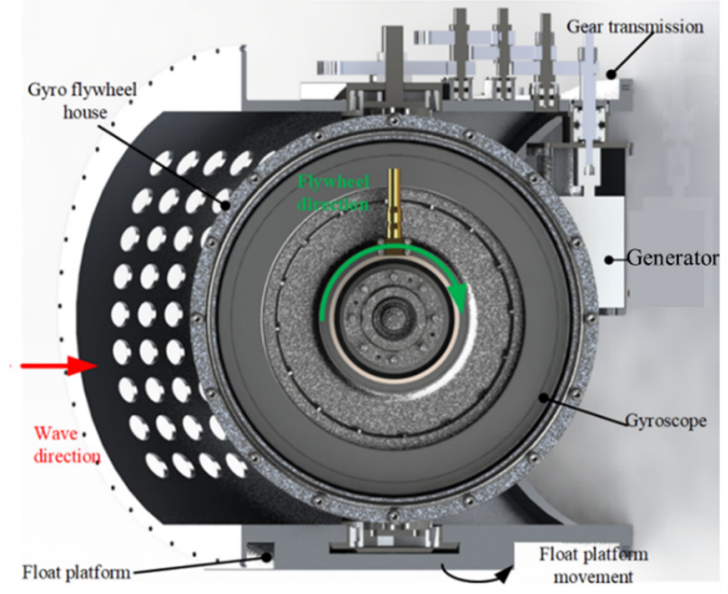

Fig. 1. Schematic of the structure of the GWEC

When the rotor rotates at a high speed, if the applied external moment is along the axis other than the rotation axis, the gyroscope does not move in the direction of the external moment, and the direction of its angular velocity is perpendicular to the direction of the action of the external moment. This characteristic is called precession of a gyroscope [7]. As shown in Fig. 2, the rotor rotates around the $\mathrm{X}$-axis at a high speed and exerts a force $\mathrm{F}$ opposite to the $\mathrm{Z}$-axis perpendicular to the $\mathrm{X}$-axis at one end of the rotor. the gyro will not rotate around the $\mathrm{Y}$-axis under the action of the force $F$, but rotates around the $\mathrm{Z}$ axis.

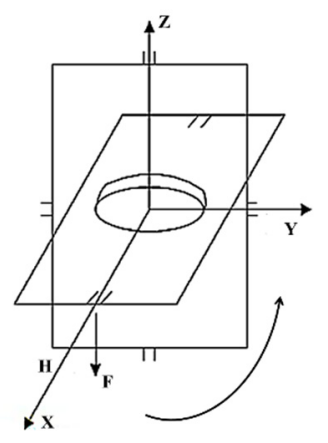

Fig. 2. Schematic diagram of gyro precession

\subsection{Mathematical model of GWEC}

Assuming that the gyro rotor rotates about the Z-axis, the floating platform and the flywheel house are both rotate around the $\mathrm{X}$-axis at an angular velocity $\omega_{x b}$, while the flywheel house rotates around $\mathrm{Y}$-axis at an angular velocity $\dot{\beta}$ [8]. Assuming that the flywheel housing coordinate system is used as the moving coordinates system (the unit vector of the moving coordinate system is $\mathbf{i}, \mathbf{j}, \mathbf{k}$ respectively), then: the angular velocity of rotation of the flywheel housing relative to the base of the float:

$$
\omega_{r}=\dot{\beta} \cdot \mathbf{j}
$$

The angular velocity of the floating body relative to the fixed space is $\omega_{x b}$, which can be decomposed into:

$$
\omega_{x b}=\omega_{x b} \cos \beta \cdot \mathbf{i}+\omega_{x b} \sin \beta \cdot \mathbf{k}
$$

So the angular velocity of rotation of the flywheel housing relative to the inertial space:

$$
\begin{aligned}
\omega & =\omega_{r}+\omega_{x b} \\
& =\omega_{x b} \cos \beta \cdot \mathbf{i}+\dot{\beta} \cdot \mathbf{j}+\omega_{x b} \sin \beta \cdot \mathbf{k}
\end{aligned}
$$

When the flywheel rotation angular velocity is $\dot{\gamma}$, thus the angular velocity of the rotor relative to the inertial space:

$$
\begin{aligned}
\omega^{\prime} & =\omega+\dot{\gamma} \cdot \mathbf{k} \\
& =\omega_{x b} \cos \beta \cdot \mathbf{i}+\dot{\beta} \cdot \mathbf{j}+\left(\omega_{x b} \sin \beta+\dot{\gamma}\right) \cdot \mathbf{k}
\end{aligned}
$$

Then the momentum moment of the rotor can be obtained (Where $J_{x}, J_{y}, J_{z}$ are the moments of inertia in $\mathrm{x}, \mathrm{y}, \mathrm{z}$ directions.):

$$
H=J_{x} \omega_{x b} \cos \beta \cdot \mathbf{i}+J_{y} \dot{\beta} \cdot \mathbf{j}+J_{z}\left(\dot{\gamma}+\omega_{x b} \sin \beta\right) \cdot \mathbf{k}
$$

According to the theorem of kinetic energy and Coriolis Effect:

$$
\frac{d H}{d t}=\frac{d \tilde{H}}{d t}+\omega \times H=M
$$

Available from Eq. (3) (6), and the variation of the precession angle is the key to wave energy generation, so the component of the y-axis is:

$$
J_{y} \ddot{\beta}+\left(J_{x}-J_{z}\right) \omega_{x b}^{2} \sin \beta \cos \beta-J_{z} \omega_{x b} \dot{\gamma} \cos \beta=M_{y}
$$

Assuming that the gyro precession axis directly interacts with the generator rotor, the generator is a DC generator and the load is a pure impedance load [9], there are:

$$
T=K_{i} \frac{K_{u}}{R_{L}} \dot{\beta}=k \dot{\beta}
$$

Among them, $K_{u}$ is the motor speed constant, $K_{i}$ is the motor torque constant, $R_{L}$ is the load resistance. So that load characteristics approximate to damping characteristics, then:

$$
T=M_{y}=k \dot{\beta}
$$

$$
\begin{gathered}
\ddot{\beta}=\left[k \dot{\beta}-\left(J_{x}-J_{z}\right) \omega_{x b}^{2} \sin \beta \cos \beta+J_{z} \dot{\gamma} \omega_{x b} \cos \beta\right] / J_{y} \\
P=M \omega=M_{y} \dot{\beta}=k \dot{\beta}^{2}
\end{gathered}
$$




\section{Maximum power control}

According to the power formula, the maximum power tracking of GWEC can be realized by changing the damping coefficient $\mathrm{k}$. And the damping coefficient $\mathrm{k}$ is related to the external load. Combining with the previous analysis, we can see that there are load resistance values corresponding to the specific sea wave amplitude to maximize the power. The control block diagram of this paper is shown in Figure 3.

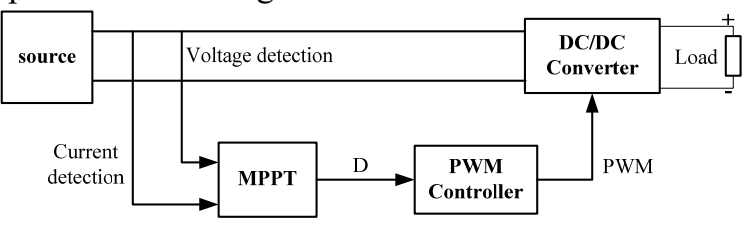

Fig. 3. Principle diagram of control system

In the figure, $\mathrm{DC} / \mathrm{DC}$ circuit is regarded as equivalent load. To adjust it is equivalent to adjusting k. Firstly the output voltage and current of gyroscopic sea wave device are sampled and the power is calculated [10]. Then a PWM signal is obtained by the maximum power algorithm: perturbation and observation (P\&O) method and incremental conductance method. The PWM signal is used to control the switching on and off of the switch in DC/DC converter circuit, thus changing the equivalent load of the circuit and realizing maximum power tracking.

The basic principle of perturbation and observation method is to apply a perturbation (such as voltage or current) at regular intervals and then observe the direction of output power to determine the direction of the next moment of perturbation [11].

Firstly, it detects the output voltage and current at the current moment, then calculates the output power at the current moment, and compares it with the output power at the previous moment. If the output power does not change, it shows that the current system has worked at the maximum power point. Otherwise, the direction of output power change is judged, and the direction of voltage disturbance at the next moment is judged according to the method described above, so that the circuit can work at the maximum power point.

The relationship between voltage disturbance and power disturbance can be approximately expressed as "positive in the same sign, negative in the different sign". That is, when they all show the same direction, the circuit will continue to change in the disturbance direction of the previous moment; when they are different, the disturbance direction of the previous moment will change in the opposite direction.

The basic principle of incremental conductance method is that when the circuit runs at the maximum power point, the derivative of power to voltage is zero, and the direction of the next cycle disturbance of the circuit can be determined by judging the magnitude of the derivative [12]. When the derivative is greater than zero, the circuit runs on the left side of the maximum power point, and the disturbance needs to be increased; otherwise, if on the right side, the disturbance needs to be reduced.

\section{Simulation result}

A simulation test platform of the two algorithms above is built by using MATLAB/Simulink. As shown in Figure 4, the input signal is a step signal with two changes. The change time points are 1 second and 2 seconds, respectively. The signal voltage increases at 1 second and decreases at 2 seconds, so as to observe whether the output power can track the change of the signal, so that the circuit can be quickly maintained near the maximum power. The simulation parameters are shown in Table 1.

Table 1. Simulation parameter

\begin{tabular}{|c|c|}
\hline Parameters & Value \\
\hline $\begin{array}{c}\text { Rotational inertia of gyro } \\
\text { rotor }\end{array}$ & $0.372 \mathrm{~kg} \cdot \mathrm{m}^{2}$ \\
\hline Rotational inertia in X/Y axis & $0.211 \mathrm{~kg} \cdot \mathrm{m}^{2}$ \\
\hline Angular velocity of gyro rotor & $10000 \mathrm{r} / \mathrm{min}$ \\
\hline Amplitude of waves & $8^{\circ}$ \\
\hline Period of waves & $7 \mathrm{~s}$ \\
\hline
\end{tabular}

The simulation time is 1 second and the simulation sampling time is 0.01 seconds. The simulation results are shown in Fig. 5 and Fig. 6, respectively. When power is not optimized, the result is shown in Figure 7.

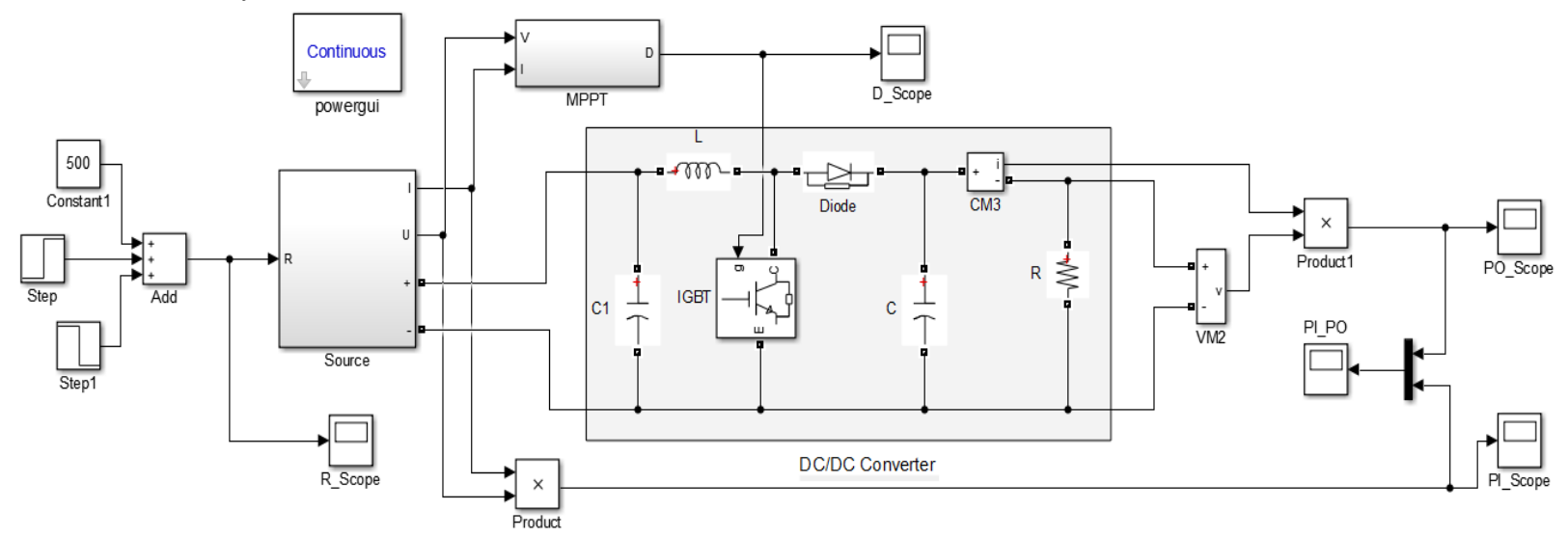

Fig. 4. Simulation diagram of MPPT 


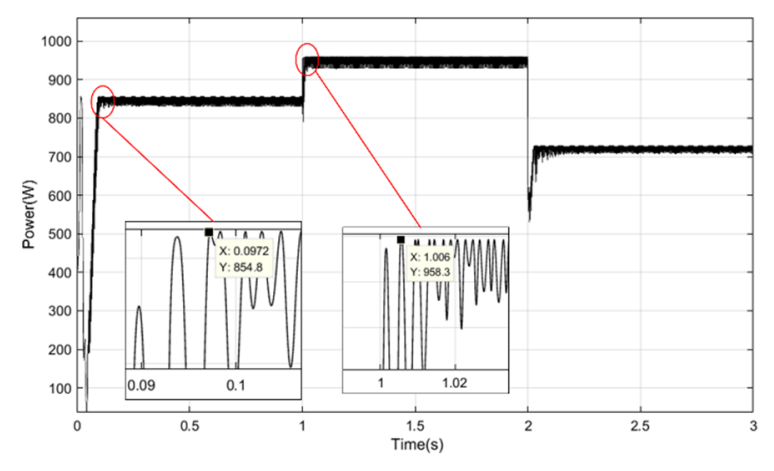

Fig. 5. Simulation result of $\mathrm{P} \& \mathrm{O}$

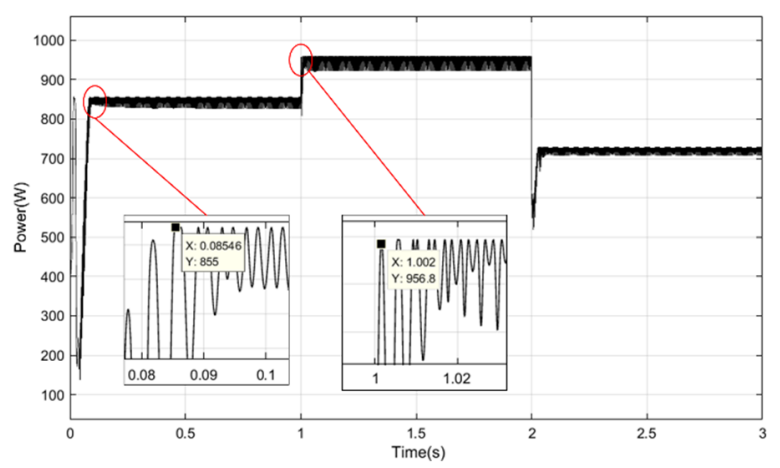

Fig. 6. Simulation result of incremental conductance method

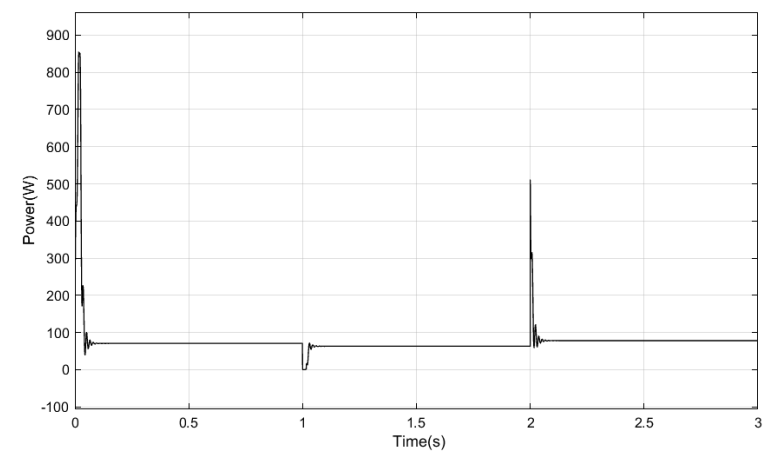

Fig. 7. Simulation result of no MPPT

From the result, When the circuit does not carry out maximum power tracking, the power cannot be maintained at the maximum power point, even will decrease a lot when it reaches the maximum power. However, when maximum power tracking is performed, both of two algorithms can keep the circuit working near the maximum power point. In contrast, the power curve by incremental conductance method has a faster tracking speed, but the fluctuation of the curve is larger; while the power curve by perturbation and observation method is not so fast, but the curve is more stable and the fluctuation is small.

\section{Conclusion}

In this paper, a novel type of gyroscopic wave energy converter is proposed and two different methods were used for comparison and verification. The simulation results show that the maximum power tracking can be achieved by perturbation and observation method and incremental conductance method when the sea wave parameters change. Generally speaking, the perturbation and observation method is more effective. The fluctuation of output power curve is small and the tracking speed is fast. In addition, the method has high accuracy and good reliability. And the output power of incremental conductance method fluctuates greatly, the tracking speed has no obvious advantages, and the accuracy and reliability are poor. The incremental conductance method only applies to relatively simple systems. Based on a new idea of using gyroscope effect to realize wave power generation, this paper carries out simulation research on it, which provides guidance and reference for the development of physical prototype.

\section{References}

1. Dai Youming, Research on a floating double-floating wave energy generating device, SCUT, (2015)

2. Yao Qi, Wang Shiming, Hu Haipeng, Development and Prospect of Wave Power Generation Devices, MDM, 33, 86-92 (2016)

3. J. V. Ringwood, G. Bacelli, F. Fusco, EnergyMaximizing control of wave-energy converters, IEEE, 34, 30-55 (2014)

4. C. Velez, Z. H. Q, K. C. Lin and S. Y. Jin, Design, Modeling and Optimization of an Ocean Wave Power Generation Buoy. MTSJ, 48, 51-60 (2014)

5. H. Mendonca, S. Martinez, A resistence emulation approach to optimize the wave energy harvesting for a direct drive point absorber, IEEE. TSE, 7, 3-11 (2016)

6. E. Amon, A. Schacher, T. K. A. Brekken, A novel maximum power point tracking algorithm for ocean wave energy devices, Proc. ECCE, 2635-2641 (2009)

7. Fan Zhongtian, Xu Fangjie, Research on the precession of gyroscope suspended under a light rope with torsion resistance, 40, 72-75 (2018)

8. Yu Fachuan, Development of a one-wheeled robot based on gyro precession effect, HUT, (2015)

9. Liu Yanzhu, Gyroscopic Mechanic, BSP, (2009)

10. M. B. Smida, A. Sakly, Genetic based algorithm for maximum power point tracking (MPPT) for grid connected PV systems operating under partial shaded conditions, IEEE, 1-6 (2015)

11. Zheng Mingyue, Research on Oscillating Float Wave Energy Generation Technology, SCUT, (2017)

12. E. Tedeschi, M. Carraro, M. Molinas, Effect of control strategies and power take-off efficiencies on the power capture from sea wave, IEEE.TEC, 26 1088-1098 (2011) 